\title{
Implementación de la manufactura esbelta en una línea de arneses eléctricos automotrices
}

\section{Implementation of lean manufacturing in an automotive electric harness line}

\author{
CANO-CARRASCO, Adolfo†*, VÁSQUEZ-TORRES, María del Carmen, GONZÁLEZ- \\ VALENZUELA, Elizabeth y BELLIZIA- GUZMÁN, Héctor
}

Instituto Tecnológico de Sonora

ID $1^{\text {er }}$ Autor: Adolfo, Cano-Carrasco / ORC ID: 0000-0002-3392-3667, Researcher ID Thomson: G-5035-2018, arXiv Author ID: adolfo.cano, CVU CONACYT ID: 266064

ID $1^{\mathrm{er}}$ Coautor: María del Carmen, Vásquez-Torres / ORC ID: 0000-0003-0938-4955, Researcher ID Thomson: X-21042018, CVU CONACYT ID: 286266

ID $2^{\text {do }}$ Coautor: Elizabeth, González-Valenzuela / ORC ID: 0000-0003-3774-5324, Researcher ID Thomson: G-5042-2018, arXiv Author ID: elizabeth_gonzalez_v, CVU CONACYT ID: 276316

ID $3^{\text {er }}$ Coautor: Héctor, Bellizia- Guzmán / ORC ID: 0000-0001-9030-3344

DOI: $10.35429 / J T O .2019 .9 .3 .21 .29$

Recibido 8 de Enero, 2019, Aceptado, 30 de Marzo, 2019

\section{Resumen}

Esta investigación analiza la implementación de la filosofía de Manufactura Esbelta a través de un caso práctico en la línea de ensamble final en una empresa elaboradora de arneses automotrices eléctricos y pretende demostrar que los desperdicios actuales del proceso productivo bajo estudio serán eliminados mediante la aplicación sostenible de la Teoría de la Manufactura Esbelta. La metodología utilizada se realizó con ayuda del círculo Deming: Planificar objetivos, línea modelo, equipos, capacitación, Ejecutar: diseñar estaciones, Reconocer desperdicio, Establecer flujo, crear lotes mixtos, calcular Tak-time, Determinar operaciones coincidentes, Determinar sistema de jalar, Verificar: el lanzamiento y Actuar: determinar verificación y seguimiento. Posteriormente se analizó los programas aplicados y las actividades utilizadas para el desarrollo de la filosofía de manufactura esbelta. La principal contribución es compartir los resultados de un caso de éxito en la implementación de la filosofía de manufactura esbelta al analizar la efectividad de los programas y actividades de soporte en su desarrollo.

Filosofía, Manufactura Esbelta, Kaizen

\begin{abstract}
This research analyzes the implementation of the Lean Manufacturing philosophy through a case study in the final assembly line in an electric automotive harness manufacturing company and intends to demonstrate that the current waste from the production process under study will be eliminated through the sustainable application of The Theory of Lean Manufacturing. The methodology used was carried out with the help of the Deming circle: Plan objectives, model line, equipment, training, Execute: design stations, Recognize waste, Establish flow, create mixed lots, calculate Tak-time, Determine matching operations, Determine pull system, Verify: launch and Act: determine verification and monitoring. Subsequently, the programs applied and the activities used for the development of the lean manufacturing philosophy were analyzed. The main contribution is to share the results of a successful case in the implementation of the lean manufacturing philosophy when analyzing the effectiveness of the programs and support activities in its development.
\end{abstract}

Philosophy, Lean Manufacturing, Kaizen

Citación: CANO-CARRASCO, Adolfo, VÁSQUEZ-TORRES, María del Carmen, GONZÁLEZ-VALENZUELA, Elizabeth y BELLIZIA- GUZMÁN, Héctor. Implementación de la manufactura esbelta en una línea de arneses eléctricos automotrices. Revista de Operaciones Tecnológicas. 2019. 3-9: 21-29

\footnotetext{
* Correspondencia del Autor (Correo electrónico: adolfo.cano@itson.edu.mx)

$\dagger$ Investigador contribuyendo como primer autor.
} 


\section{Introducción}

No obstante que las técnicas, métodos y filosofías de mejora continua ofrecen muchas ventajas para las organizaciones. En México es poca le evidencia empírica acerca de investigaciones que utilicen estadísticas descriptivas o análisis multivariados que muestren el nivel de su adopción en plantas mexicanas. (Monge y Cruz, 2015).

En una investigación realizada en el sector de Apodaca Monge y Cruz (2015) obtuvieron s resultados que coinciden con lo establecido por Liker (2011) y Bowen (1999) en la que el uso de herramientas y técnicas de manufactura lean fue implementado de forma aislada y no consistente produciendo como resultado un programa insostenible de la filosofía lean (Liker et al, 2011; Spear y Bowen, 1999). Un hecho relevante es que las empresas de todo el mundo se jactan de los resultados que han conseguido por sus programas Lean sin embargo, a pesar de todos los proyectos realizados, ninguna compañía en ninguna industria en el mundo ha alcanzado el nivel de excelencia operacional que Toyota ha logrado (Liker y Convis, 2011).

Por otra parte (Klochkov, Gazizulina y Muralidharan, 2019) señalan que el objetivo de implementar la manufactura esbelta varía desde la organización del control y las pruebas hasta la optimización de los esquemas logísticos, siendo enfocado para reducir los costos de producción, mejorar la confiabilidad del proceso, reducir el ciclo de producción y garantizar la calidad, por lo que es requerido tener objetivos establecidos, plazos, asignación de responsabilidad y autoridad, requisitos para determinar riesgos y mantener registros implicando tener un liderazgo empresarial sólido, conocimiento de mano de obra y tecnología.

En este sentido un primer aspecto a considerar para el éxito en la aplicación de la manufactura esbelta es la visualización del largo camino que implica el llegar a una implementación exitosa, la excelencia operacional implica el desarrollo de un proceso profundo y paciente en el cual se involucra los distintos niveles de la organización (Liker y Convis, 2011).
Esta investigación se llevó acabo en una empresa dedicada a la elaboración arneses automotrices en la línea de ensamble final, en dicha línea la materia prima es surtida (de almacén) por los manejadores de materiales a la ubicación que le fue asignada dentro de la línea en donde coloca todos los componentes necesarios para la construcción del arnés como son: Circuitos, Conectores, Mallas, Tape etc.

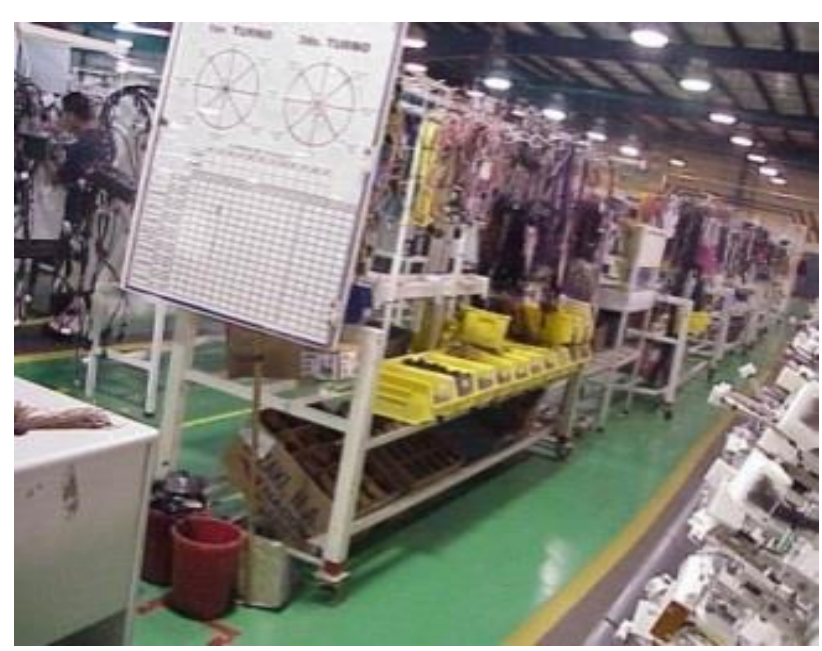

Figura 1 Espacio ocupado en líneas por materia prima

Sin embargo la ubicación la materia prima ocupa gran espacio en las líneas de ensamble final (véase figura 1), los inventarios son altos (véase figura 2), así como los costos por mantener stock, el tener demasiado surtido de material en la línea puede provocar daños y problemas que ponen en riesgo la calidad del producto.

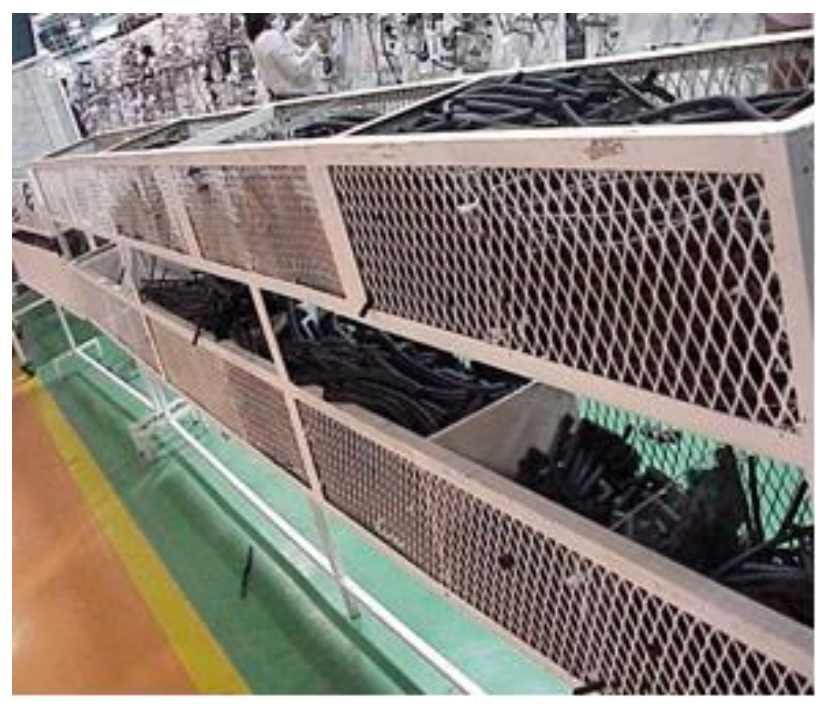

Figura 2 Niveles de stock en líneas

En lo que respecta al ensambles de circuitos éstos se van arrastrando dentro y fuera de la línea de ensamble final, los cuales pueden enredarse, ensuciarse, e incluso dañarse. 
En las estaciones de ensamble por lo general cuando se va a tomar un circuito, éste se enreda con los demás circuitos ya que los mazos de circuitos colocados en el rack se componen de entre 50 y 100 circuitos por mazo, ésta es una operación en la que se detectan más lapsos de tiempos muertos debido a que se ensamblan con rapidez un gran número de circuitos o conectores sin respetar el flujo por estación (número de piezas permitidas por estación de trabajo).

En la operación de misceláneos los rollos de tape son tomados de los contenedores en los que llega la materia prima (en algunas ocasiones) o de las grandes charolas ubicadas en la línea (ver figura 3), dentro de la misma y donde se lleva a cabo la transformación de la materia prima.

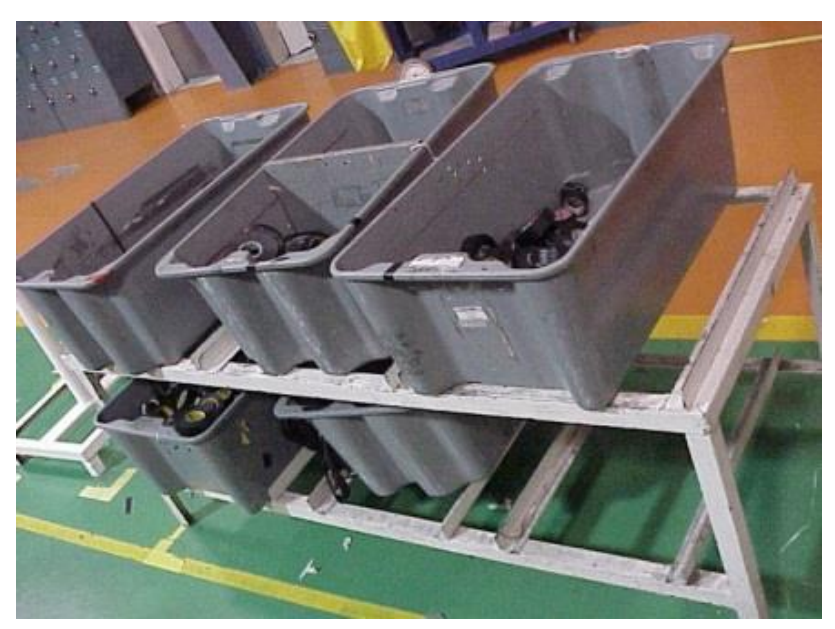

Figura 3 Ubicaciones para rollos de tape

Existen operaciones complejas ya que el diseño del tablero no permite al operador realizar con facilidad la operación o estas requieren una mayor habilidad, por lo que cuando un operador tiene asignada una operación clave y llega a faltar no es fácil cubrir este hueco, la mayoría de las ocasiones se cubre con cualquier operador y no se informa al inspector de control de calidad para tener un mayor cuidado con los defectos que se pudieran generar durante el turno.

Por otra parte al momento de una auditoria no hay un sistema que señale cuáles son las operaciones claves, y no existe un documento en donde se pueda ver que operación realiza cada operador, y en caso de que exista algún tipo de problema en una línea solamente se enteran los involucrados que se encuentran más cerca de la problemática.
Los operadores no cuentan con un área delimitada dentro de cada estación de trabajo y esto se refiere al atraso o adelanto de su ejecución, por lo que no se conoce cuando un operador se encuentra trabajando en las condiciones normales de trabajo, o si tiene tiempo de ocio.

Gracias a las normas de certificación cada operador, trabaja bajo procedimientos e instrucciones de trabajo, sin embargo, la mayoría de los trabajadores ni siquiera los conocen debido a que se encuentran en una carpeta donde solamente tiene acceso el supervisor.

Uno de los recursos más valiosos para las empresas hoy en día son "los operadores", por lo tanto es necesario enfocarse a las operaciones que realizan y como lo hacen para determinar la mejor opción en cuanto a las operaciones que desarrollan, así como la seguridad del personal, que tipo de riesgos sufre, si la estación de trabajo está diseñada de tal manera que les permita realizar adecuadamente su trabajo o qué tipo de modificaciones tienen que realizarse para lograr una mejora, si es que hacen movimientos innecesarios, lo que significa ir por materia prima lejos de la estación de trabajo.

\section{Problema}

"Existencia de operaciones que no agregan valor y desperdicio en la línea de ensamble final”.

\section{Objetivo}

Implementar la filosofía de manufactura esbelta en una línea de ensamble final de una empresa manufacturera de Cd. Obregón, Sonora, para eficientar su operación.

Hipótesis: Los desperdicios actuales del proceso productivo bajo estudio serán eliminados mediante la aplicación sostenible de la Teoría de la Manufactura Esbelta.

\section{Marco teórico}

La manufactura esbelta es una filosofía derivada del sistema de producción Toyota, y se fue acuñando gracias a las aportaciones de Kichiro Toyoda, Eiji Toyoda y Taiichi Ohno. 
Las principales aportaciones que fueron conformando esta filosofía fueron en un primer momento la creación de un sistema de "eliminación completa de todos los residuos" y la búsqueda de métodos más eficientes en la producción, posteriormente el desarrollo de una filosofía basada en el "justo a tiempo", la cual representa uno de los pilares básicos del sistema de producción Toyota y retomando el trabajo $\mathrm{W}$. Edwards Deming se desarrolló el "método de control de calidad", basado en la mejora tecnológica de cada etapa de un negocio logrando así la puesta en práctica de los principios de justo a tiempo y Kaizen, dirigidos al aumento de la productividad Ohno (1988).

El término Lean o Esbelto fue utilizado por Womack y Jones (2003) como un descriptor de la puesta en práctica de los cinco principios bajo los cuales se rige esta filosofía un constante enfoque a la creación del valor, una visión de proceso a través de la cadena de valor, establecer el flujo, jalar la producción para control y la búsqueda de la perfección.

Es así como una organización que pretende poner en práctica este pensamiento esbelto debe comenzar con un intento consciente de definir con precisión el valor en términos de productos específicos con capacidades específicas ofrecidas a precios específicos a través de un diálogo con clientes específicos.

De este modo la manufactura esbelta representa un sistema de producción el cual tiene como meta trabajar con menos recursos, menos desperdicio, menos esfuerzo humano, menos espacio para la operación, menos inversión en herramientas y menos complejidad para desarrollar nuevos productos (Alireza, Yusof and Seyed, 2011).

Alireza et al (2011) argumenta que lo que distingue a la manufactura esbelta de otras metodologías de mejoramiento es que esta se enfoca en el flujo de valor a lo largo de toda la cadena de producción mientras que otros son más específicos, otras metodologías se enfocan a los procesos de mayor valor agregado mientras que la manufactura esbelta lo hace enfocándose en las actividades que no agregan valor.
El impacto de la manufactura esbelta apunta a distintas áreas organizacionales las cuales contribuyen a la sostenibilidad tal como lo expone Galdino, Gomes y Toledo (2017) en una investigación sobre el estado del arte, siendo las principales la reducción de costos, el incremento de la calidad del producto, la reducción de la variabilidad del proceso, la aceleración del tiempo de entrega, la reducción de la tasa de defectos, la reducción de desperdicio, el incremento en la satisfacción del cliente, la reducción del tiempo de ciclo, el incremento en la satisfacción del empleado, el incremento en la calidad del servicio, reducción del tiempo de procesos, reducción de tiempo de espera y la reducción de los niveles de almacenamiento.

De igual forma Cherraf, Elfezazi, Chiarini, Mokhlis, y Benhida, en el (2016) mencionan como principales técnicas dentro de la filosofía la aplicación de las 5S, el Kaizen, el mapeo de procesos de valor (Value Stream Mapping), el sistema de jalar (Kan ban/pull), la Manufactura celular, el Mantenimiento total productivo, la reducción de tiempo de preparación (SMED), la relación con el proveedor, los mecanismos a prueba de error (pokayoke), Six sigma, Control estadístico de proceso, Administración visual (Andon), Herramientas de análisis (5Why's, Diagrama de Pareto, Diagrama causa y efecto etc.), Trabajo estándar y Distribución de planta.

\section{Método}

Sujetos: El personal participante en este proyecto tuvo una edad promedio de 25 años, nivel académico en su mayoría de preparatoria en el área administrativa y en el área operativa nivel primaria. Los roles implicados fueron: Gerente de Ing. Industrial, Superintendente de Ing. Industrial, Gerente divisional, Gerente de Calidad, Superintendente de calidad, Supervisor de control de calidad, supervisores y operadores de línea.

Las actividades relativas a la implementación se distribuyeron en las cuatro fases del circulo Deming el total de actividades consideradas es el siguiente: 1) Capacitar en la cultura de la manufactura esbelta, 2) Definir línea modelo a través de la identificación de las actividades y su distribución física, 3) Definir objetivos al implementar la cultura, 4) Establecer y capacitar equipos para implementación actividades.

CANO-CARRASCO, Adolfo, VÁSQUEZ-TORRES, María del Carmen, GONZÁLEZ-VALENZUELA, Elizabeth y BELLIZIA- GUZMÁN, Héctor. Implementación de la manufactura esbelta en una línea de arneses eléctricos automotrices. Revista de Operaciones Tecnológicas. 2019 
6) Definir estaciones de trabajo 7) Reconocer el desperdicio en el proceso, 8) Establecer el flujo de producción a través de la producción nivelada, crear lotes mixtos, calcular tiempo de alistamiento reducido y determinar operaciones coincidentes 9) Determinar sistema de jalar, 10) Lanzar la línea modelo, 11) Planear actividades para la verificación y seguimiento.

\section{Resultados}

Como parte de la planificación del proyecto se plantearon los siguientes objetivos.

1. Establecer un flujo continuo en las operaciones del proceso.

2. Eliminar el desperdicio en el proceso.

3. Mejorar a través de eventos kaizen las estaciones de trabajo.

4. Establecer tableros para monitoreo y control de indicadores de seguimiento.

Un layout de la línea de ensamble final elegida como línea modelo para implementación de la filosofía se muestra en la fig. 4.

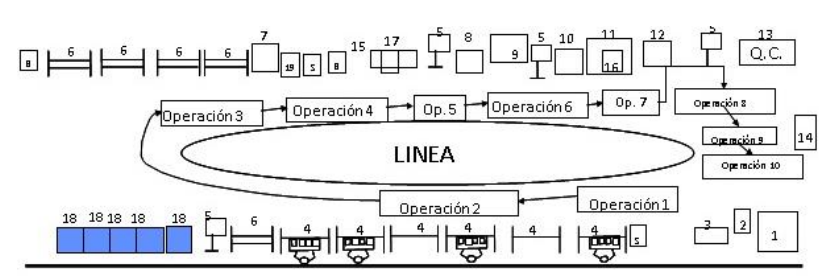

Figura 4 Lay out de la línea modelo antes de la implementación de la Manufactura Esbelta

En la figura 4 se pueden observar los componentes de una línea normal de producción, así como el flujo que sigue el proceso y por las estaciones que pasa para ser transformado en el producto terminado (arnés).

Las áreas incluidas en la distribución antes de aplicar la manufactura esbelta incluyen 1) Área de empaque, 2) Área de amarres, 3) Escritorio para supervisor de producción, 4) Rack para cablea y componentes, 5) Caballete para gráficos, 6) Rack para misceláneos, 7) Centro de carga de la línea, 8) Locker para material de alto valor, 9) Rack para cover, 10) Computadora, 11) Expansora, 12) Inspección de fusibles, 13) Mesa de inspección, 14) Mesa de amarres, 15) Recipientes vacíos, 16) Solidos impregnados, 17) Tablero auditoría integral, 18) Banco de circuitos.

\begin{tabular}{|l|r|r|}
\multicolumn{2}{|c|}{ Operadores } & \multicolumn{1}{l|}{ Oespués } \\
\hline Operadores & Antes & \multicolumn{1}{l|}{. } \\
\hline Pre ensamble & 8 & 6 \\
\hline Ensamble & 14 & 13 \\
\hline Encinte & 2 & 1 \\
\hline Prueba eléctrica & 2 & 1 \\
\hline Visual & 1 & 1 \\
\hline Empaque & 1 & 2 \\
\hline Amarres & 1 & 1 \\
\hline Terminado de arnés &
\end{tabular}

Tabla 1 Cantidad de operarios antes y después

Después de redistribuir los espacios y aplicar los principios de balancear operaciones se tuvo una reducción de 35 a 29 operadores ver tabla 1.

Así mismo la nueva distribución fig. 5 se compactó a las áreas siguientes 1) área de empaque, 2) área de amarre 3) centro de carga de la línea 4) computadora 5) Expansora 6) Inspección de fusible 7) Mesa de amarre 8) Mesa de amarre 9) Auditoria integral 10) Banco de circuitos 11) Preformado 12) Carrito surtido y 13) Información.

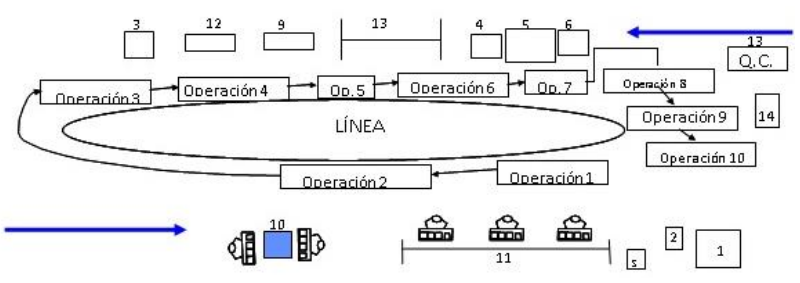

Figura 5 Lay out de la línea modelo después de la implementación de la Manufactura Esbelta

Computadora bar code, 5) Expansora, 6) Inspección de fusible, 7) Mesa de inspección, 8) Mesa de amarre, 9) Tablero de auditoría integral, 10) Banco de circuitos, 11) Mesa de preformado, 12) Carrito de surtido, 13) Información.

En la línea se producen los modelos de arneses A, B y C Considerando 516 min/día de disponibilidad en el turno y una demanda diaria de 240 u de modelo A, 120 de modelo B y 60 de modelo $\mathrm{C}$, se calculó el tak time para cada modelo a producir en la línea (tiempo asignado para la producción de una unidad en minutos o segundos es calculado con el tiempo disponible considerando las horas oficiales de trabajo, menos descanso y comida).

Tak time $=\frac{\text { tiempo disponible }}{\text { demanda }}$

Tak time $=\frac{516}{240}=2 \mathrm{seg} / \mathrm{u} \quad$ Para modelo A de igual modo resultan $4 \mathrm{seg} / \mathrm{u}$ para B y $8 \mathrm{seg} / \mathrm{u}$ y C. 
La corrida de producción normal es: $A A A A A A A A B B B B C C$ lo que representaría un lote mixto de producción como $A A B A A B C A A B A A B C$.

Debido a los cambios en el diseño de las estaciones fue necesario modificar la secuencia de operaciones, para elevar la productividad se realizó una asignación multi-procesos considerando el Tak time y las operaciones coincidentes ver fig. 6 y una vez que se logró nivelar la producción el siguiente paso es establecer el flujo de producción, para el logro de éste fue necesario realizar un flujo ininterrumpido de producto, esto significa una pieza después de otra, creando un marco de trabajo sincronizado en todos los procesos (ver anexo 15) ver figura 6.

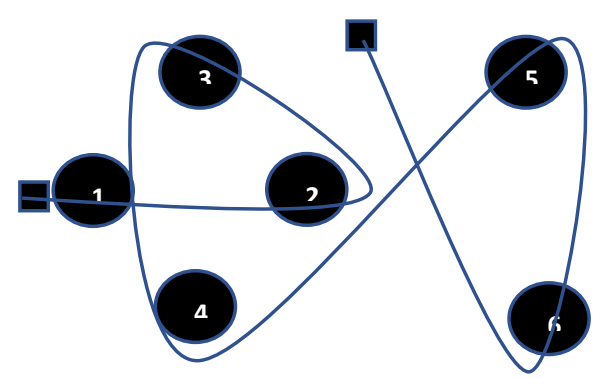

Figura 6 Operaciones coincidentes

Además se delimitaron estación de trabajo, y se mejoró el flujo de materiales a través de estas (ver figura 7).

1)

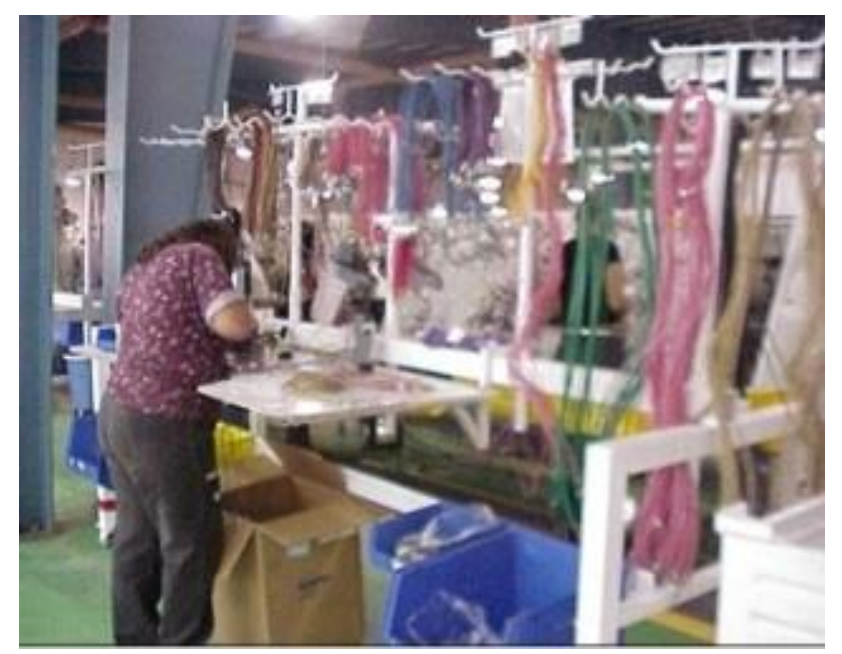

Figura 7 Kaizen de estación de trabajo antes de la implementación

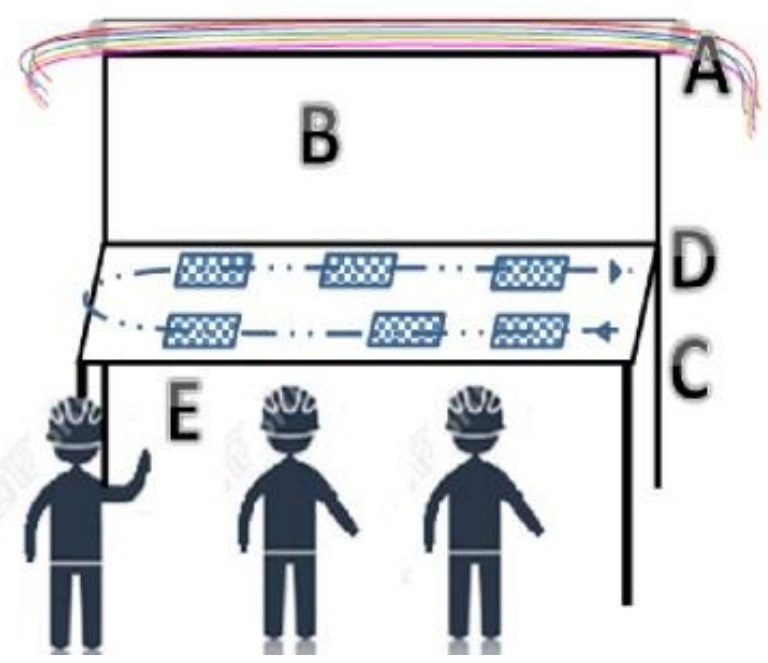

Figura 8 Kaizen de estación de trabajo después de implementación

La imagen 7 muestra una estación de preformado del proceso antes de la implementación de la manufactura esbelta, en donde las charolas de colores azul y amarillo contienen los componentes y en la parte superior los circuitos montados en una estructura de metal. Por otra parte la figura 8 presenta la conformación de la estación de preformado para las 3 primeras operaciones mejorada en la que el surtido de circuitos se realiza desde el área superior $\mathrm{A}$, el flujo de componentes corre de desde $C$ hasta $\mathrm{D}$ pasando a través de tres operaciones en la sección E teniendo en la sección B un soporte de ayuda visual de las operaciones. Se disminuyó el nivel de inventario con nuevos dispositivos e abastecimiento ver fig. 9 y 10.

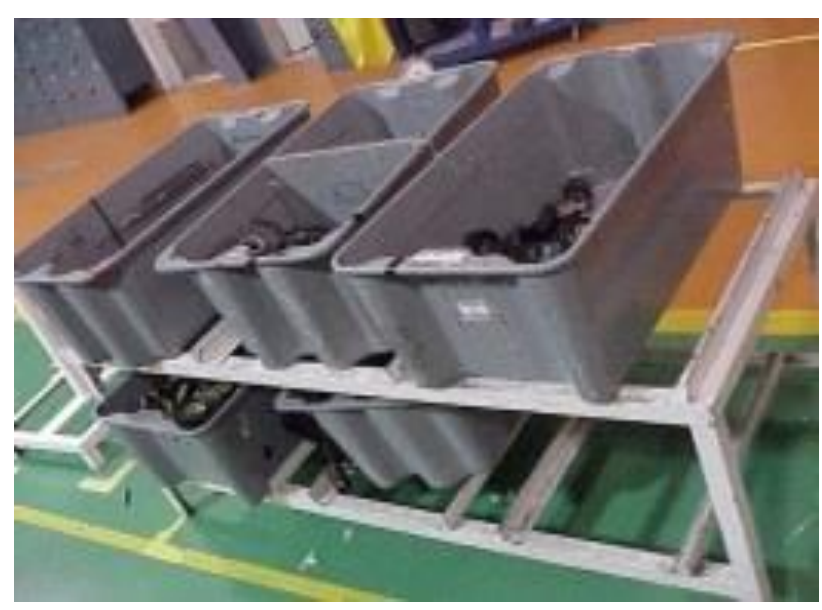

Figura 9 Kaizen inventario antes de la implementación 


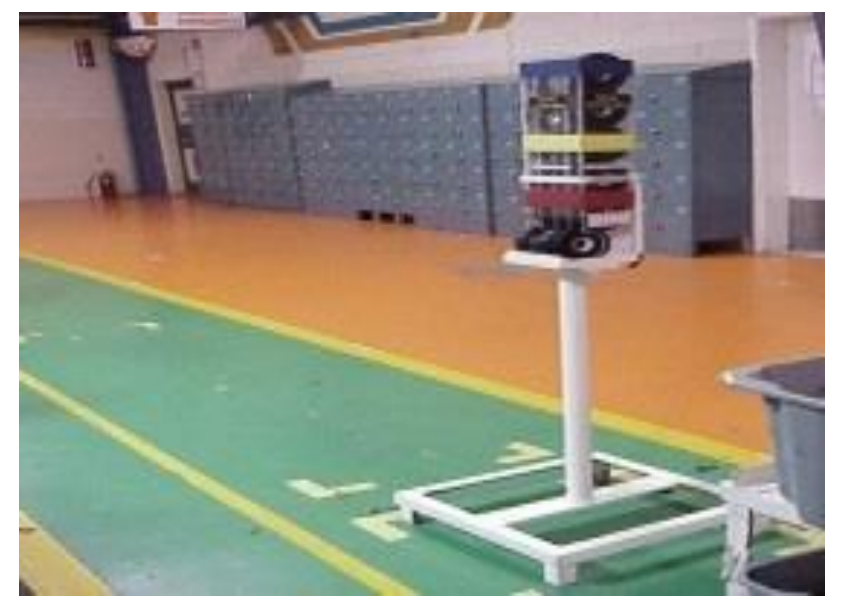

Figura 10 Kaizen de estación de trabajo después de la implementación

También se mejoró el manejo de materiales al implementar cambios en la forma de proporcionar los materiales a la vez que se redujo el nivel de inventario de estos ver figura 11 y 12 .

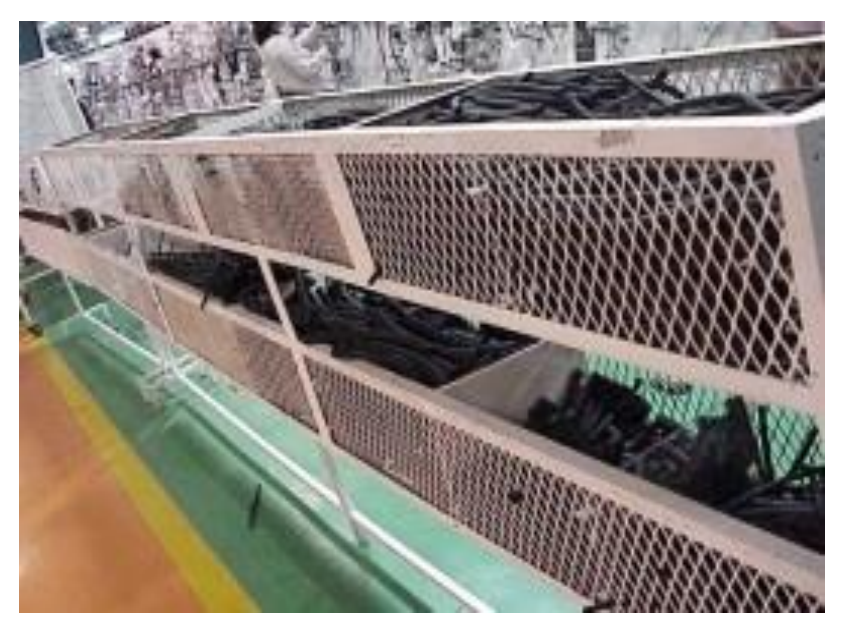

Figura 11 Kaizen de manejo de material antes de la implementación

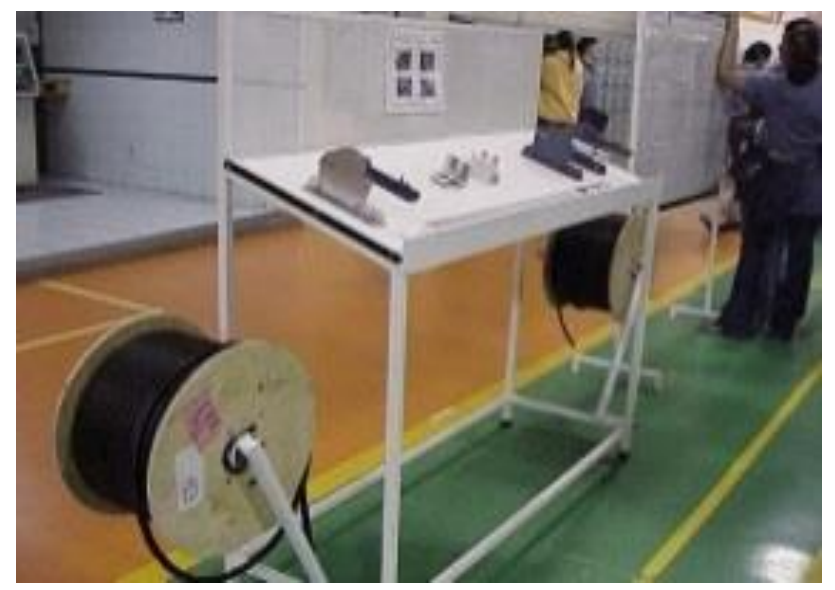

Figura 12 Kaizen de manejo de material después de la implementación
Otro aspecto relevante fue la utilización de ayuda visual en las estaciones a través de hojas de especificaciones técnicas y la implementación de un sistema de señales de andon para administrar los paros ver figura 13.

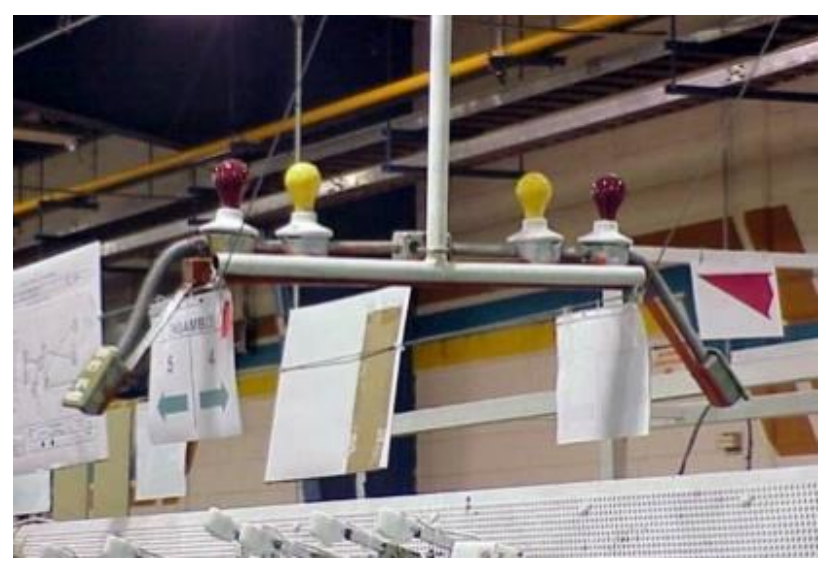

Figura 13 Sistema andon

El entrenamiento que recibió el personal de la línea fue cruzado multi-hábil ver tabla 2 , para lograr un flujo rápido y sin movilidad de material.

\begin{tabular}{|c|c|c|c|c|}
\hline $\mathbf{A}$ & B & C & D & $\leftarrow$ Multi máquinas \\
\hline 1 & 1 & 1 & 1 & \\
\hline 2 & 2 & 2 & 2 & \\
\hline 3 & 3 & 3 & 3 & \\
\hline 4 & 4 & 4 & 4 & $\uparrow$ Multi operaciones \\
\hline 5 & 5 & 5 & 5 & \\
\hline
\end{tabular}

Tabla 2 Matriz de asignación de capacitación

Debido a que en la asignación multimáquinas presenta dos tipos de problemas que son: 1) Las demoras Ínter- procesos, estas no podrían ser eliminadas, como consecuencia se ve afectado tanto el flujo en el proceso como el tiempo de entrega; 2) Aun si el Tak time es aplicado a este sistema la carga de operación del personal no puede ser ajustada rápidamente si hay un cambio en las tendencias de venta.

Lo anterior es argumento de la asignación multiprocesos considerando un layout con disposición de máquinas acorde a secuencia del proceso, un flujo ininterrumpido de producto (lote de transferencia unitario), un marco de tiempo de trabajo sincronizado y estándar, una asignación de personal para procesos múltiples y un entrenamiento cruzado (fuerza de trabajo multi-hábil) y un diseño del trabajo para que el personal pueda moverse para crear el flujo de producción. 
Se implementó un sistema Kanban el cual considera la siguiente información; 1)"Qué se produce y cuanto", 2) cuando el kanban es removido o tiempo de iniciar producción, 3) La prioridad de producción es establecida en el orden en el cual el kanban llegó.

El kanban funciona como orden de producción y control visual, para clarificar la siguiente información: 1) Grado de conformidad con operaciones coincidentes, 2) Capacidad actual del proceso, 3) Niveles de existencias actuales en el proceso, 4) Niveles apropiados de mano de obra en el proceso, 5) La situación de producción al final del proceso y 6) la urgencia de necesidades de material en el final del proceso.

Las reglas de kanban de órdenes se establecieron como 1) el volumen de producción debe coincidir con el volumen indicado en el kanban removido y la orden de producción es el orden en que los kanban fueron removidos, 2) Kanban debe viajar siempre con las partes. Las Reglas para kanban de retiro: 1) El kanban es removido del contenedor de partes exactamente cuando la primera parte es sacada del contenedor, 2) El kanban removido es llevado hacia el inicio del proceso para jalar partes, 3) El retiro de kanban es intercambiado con el kanban de orden de producción en el almacén de la parte inicial del proceso 4) Partes sin tarjetas de kanban no deben ser transportadas.

Es importante administrar el sistema con las siguientes consideraciones: 1) no usar kanban junto con el plan de producción, el plan de producción es proveído solo para la primera estación de ensamble final, 2) siempre trate de minimizar el número de kanban, 3) Transfiera kanban frecuentemente y trate de mantener las transferencias en volúmenes pequeños, 4) inicie usando kanban solamente dentro de la compañía y desde el proceso final, después de que esté establecido internamente pueden desplegarse a través de la cadena de proveedores, 5) Evite usar un kanban "todo incluido" en las fases iniciales.

La figura 10 ilustra la conformación del Kan ban para la línea de ensamble final el lote de procesamiento es unitario y el mismo Kanban hace las veces de producción y retiro.

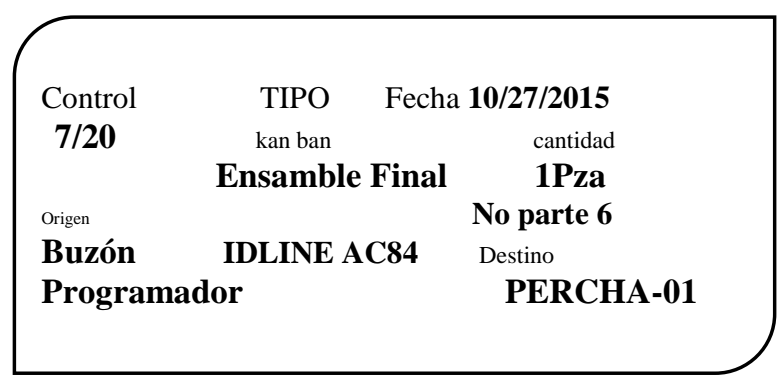

Figura 10 Kan ban de ensamble final

Para el lanzamiento de la línea modelo fue necesario diseñar los formatos para registro de datos de la operación, construir los tableros alusivos a aspectos críticos del proceso y del programa de manufactura esbelta, las siguientes actividades fueron establecidas para este propósito: 1) Identificación de operaciones, 2) Sistema automático de paro de línea cada tres estaciones, 3) Hoja de trabajo estandarizado, 4) Determinación de operaciones difíciles, 5) Diseño de estación de subensamble, 6) Hoja de auto evaluación, 7) Sistema cero defectos, 8) Tablero de medibles, 9) Tablero QCD, 10) Tablero de soluciones Kaizen, 11) Tablero GEMBA, 12) Plan de actividades para la verificación, 13) Auditoría al proceso, 14) Acciones correctivas y 15) Flujo ininterrumpido de producto.

Cada equipo de trabajo se encargó de realizar un tablero para para dar seguimiento al proceso y a la par dar cumplimiento a los principios de la filosofía de manufactura esbelta. El tablero de control de la implementación del programa $5 \mathrm{~S}$, el cual pretende monitorear los resultados de la implementación de dicho programa a través de la auditoria a las áreas teniendo como indicador el porcentaje de cumplimiento de requisitos, los cuales se dividen en cada una de las $\mathrm{S}$ del programa.

Un tablero de control QCD (quality, cost, delivery), referente a la Calidad, Costo y Entrega el cual mide el desempeño de la línea en relación a Scrap, Defectos, Costo, Tiempo de entrega de órdenes y control estadístico de variables críticas para la calidad. Un tablero Gemba, el cual permite el control de incidencias en el área de trabajo, en él se monitorea los cambios que se susciten en las 4M, Materiales, Métodos, Mano de obra y Maquinas, cada cambio es controlado para mantener los requisitos de calidad establecidos en cada uno de estos cuatro rubros. 
Por último un tablero para monitoreo de Cero defectos en el cual se registra una bitácora de las acciones correctivas asociadas a defectos registrados durante el proceso. Por último un tablero de solución para operaciones difíciles en el cual se exponen las áreas de oportunidad identificadas en el proceso y el kaizen establecido para resolverla. Todo lo anterior arrojará información relevante para la toma de decisiones durante la operación de la línea de ensamble final.

\section{Conclusiones}

Por los resultados presentados se puede argumentar que se logró implementar la filosofía de manufactura esbelta a lo largo de la línea de ensamble final, por lo cual es posible afirmar la hipótesis inicial por el hecho de que la implementación exitosa de la Manufactura esbelta, condujo a que los desperdicios actuales del proceso productivo bajo estudio fueran eliminados mediante la aplicación sostenible de la Teoría de la Manufactura Esbelta, aun que el proyecto se encuentra en la etapa inicial la ejecución a través de una metodología de mejora continua como el circulo Deming garantiza su sostenibilidad y permitió mostrar resultados significativos en el incremento de la productividad en el proceso, al ser tratado con herramientas de manufactura esbelta. Sin perder de vista el sentido holístico de esta filosofía, la experiencia de la línea modelo marca el punto de partida para migrar a un nivel superior que incluya de forma integral la operación de la empresa.

Aunque el sistema de producción pase a lotes más pequeños, aunque eliminamos los malos pasos de la operación, y aun que se tengan los indicadores clave de rendimiento mostrando mejoras que nunca pensamos que fueran posibles, Las malas noticias suelen presentarse, a medida que pasa el tiempo, los procesos parecen volverse contra sí mismos y degradarse, con la variabilidad y el desperdicio creciendo nuevamente. La sostenibilidad de la manufactura esbelta a largo plazo depende de una amplia variedad de factores tales como el involucramiento de la administración en el programa, la resistencia al cambio, la comunicación, la infraestructura organizacional, el entrenamiento y la capacitación, el enfoque al cliente, el enfoque hacia la relación con el proveedor, el factor humano, el entendimiento de técnicas y herramientas para el mejoramiento, habilidades para el manejo de los proyectos etc.
Por ello la mejora continua en cualesquier organización es una tarea constante, así tengamos décadas operando con la filosofía de manufactura esbelta estamos expuestos al fracaso.

\section{Referencias}

Alireza A., Yusof I. and Seyed M. H. H., (2011). A Study on Total Quality Management and Lean Manufacturing: Through Lean Thinking Approach. World Applied Sciences Journal 12 (9): 1585-1596.

Cherraf A., Elfezazi S., Chiarini A., Mokhlis A., Benhida K., (2016).The integration of lean manufacturing, Six Sigma and sustainability: A literature review and future research directions for developing a specific model. Elsevier, Journal of Cleaner Production 139 (2016) $828 \mathrm{e} 846$.

Galdino de Freitas J, Gomes C. H., Toledo F. F., (2017). Impacts of Lean Six Sigma over organizational sustainability: A survey Study. Elsevier, Journal of Cleaner Production 156 (2017) $262 \mathrm{e} 275$.

Liker, J y Convis, G., (2011). The toyota way to lean leadership, CRC Press, New York.

Monge C. y Cruz J., (2015). Manufacturing and continuous improvement performance level in plants of mexico; a comparative analysis among large and medium size plants. European Journal of Business and Economics, VOLUME 10, ISSUE 2.

Ohno, T., (1988). Toyota production system: Beyond large-scale production. Portland: Productivity Press.

Womack J. and Jones T. D (2003). Lean Thinking. Banish waste and créate wealth in your corporation. FREE PRESS. New York. 